\section{The effect of aspirin and warfarin therapy in trabeculectomy}

CJ Cobb'1, S Chakrabarti' ${ }^{1}$ V Chadha ${ }^{2}$ and R Sanders ${ }^{2}$
'Department of Ophthalmology, Ninewells Hospital and Medical School, Dundee, UK

${ }^{2}$ Department of Ophthalmology, Queen Margaret Hospital, Dunfermline, UK

Correspondence: V Chadha, 11 West Holmes Place, Broxburn,

West Lothian EH 52 5NJ, UK Tel: + 44786508 1415;

E-mail: vchdh@

aol.com

Received: 20 June 2005 Accepted in revised form: 23 December 2005 Published online: 3 March 2006

We have no proprietary interests in any of the products mentioned in the manuscript. No funding was obtained for this study

\begin{abstract}
Aim The management of patients on antiplatelet and anticoagulation therapy (APACT) in glaucoma surgery currently has no specific recommendations. We aimed to establish the risk of haemorrhagic complications and surgical outcome in patients on APACT in glaucoma surgery. Methods We retrospectively examined 367 consecutive trabeculectomies performed between 1994 and 1998. Preoperatively 60 $\mathbf{( 1 6 . 4 \% )}$ patients were on APACT (55 on aspirin and five on warfarin). The incidence of hyphaema and haemorrhagic complications between patients with and without APACT was documented. Surgical success was defined in two categories as an intraocular pressure (IOP) $<21 \mathrm{mmHg}$ and an IOP $<16 \mathrm{mmHg}$ 2 years following trabeculectomy with and without antiglaucoma medication.

Results None of the patients on aspirin suffered significant intra or postoperative haemorrhage. Aspirin was associated with a significantly higher risk of hyphaema $(P=0.0015)$ but this was not found to significantly affect IOP control at 2 years. Patients on warfarin suffered haemorrhagic complications and trabeculectomy failure.

Conclusions Aspirin appears to be safe to continue with during trabeculectomy. Patients on aspirin have an increased risk of hyphaema following trabeculectomy. This however does not appear to affect surgical outcome.

Warfarinised patients are at risk of serious bleeding complications. They require careful monitoring pre- and postoperatively and are at risk of trabeculectomy failure.

Eye (2007) 21, 598-603. doi:10.1038/sj.eye.6702277; published online 3 March 2006
\end{abstract}

Keywords: anticoagulation therapy; antiplatelet therapy; trabeculectomy; failure rate; hyphaema

\section{Introduction}

Aspirin is increasingly being recommended as prophylaxis against thromboembolism. ${ }^{1}$ Currently there are no agreed guidelines on the pre- and postoperative management of patients on APACT therapy in ophthalmic surgery. Cataract surgery does not seem to have an adverse outcome in patients on APACT. ${ }^{2-4}$ Despite this a proportion of ophthalmic surgeons discontinue such therapy before cataract surgery resulting in serious systemic thromboembolic complications. ${ }^{2,4}$ Parkin and Manners ${ }^{5}$ showed considerable variation in practice among consultant ophthalmologists regarding stopping and continuing with APACT therapy during oculoplastic surgery. In their study, half of the surgeons questioned would consider stopping warfarin before oculoplastic procedures and over half the surgeons had experienced complications relating to both stopping and continuing with aspirin or warfarin therapy. Flaxel and Blach ${ }^{6}$ reported on three cases that suffered significant intraoperative haemorrhage during vitreoretinal surgery, while taking aspirin.

Bell et $a l^{7}$ have reported a higher concurrence of aspirin use in patients with glaucoma who required surgical management compared with patients having relatively medically stable glaucoma. They postulated that a possible explanation for this was that patients who underwent glaucoma surgery had more systemic vascular disease. There is little data available on the effect of anticoagulation therapy in glaucoma surgery. McCormack showed that in a series of five trabeculectomies in anticoagulated patients, three developed postoperative hyphaema. ${ }^{8}$ Surgical outcome was not studied. This is of obvious importance as patients undergoing glaucoma surgery are often elderly with bilateral visual impairment and systemic comorbidity. A significant haemorrhagic operative complication may have 
a profound effect on an already compromised glaucomatous eye. On the other hand stopping APACT preoperatively may place the patient at risk of lifethreatening arterial embolism. ${ }^{2,4}$

In addition to this glaucoma surgery is now no longer a single entity. It may be combined with cataract surgery, application of intra- and postoperative antimetabolites and bleb intervention. The risk of haemorrhagic complications may predetermine the planned choice of glaucoma procedure.

We therefore carried out a retrospective analysis of a series of consecutive trabeculectomies. We aimed to compare haemorrhagic complications and the incidence of hyphaema in the early postoperative period in patients with and without APACT. We also analysed surgical outcome 2 years following trabeculectomy, in those with and without early hyphaema and in those with and without APACT.

\section{Methods}

A retrospective case note review was carried out at Ninewells Hospital, Dundee and Queen Margaret Hospital, Dunfermline from 1994 to 1998. All trabeculectomies carried out during this time period were identified from the computerised surgical logs and were carried out by nine general consultant ophthalmologists and higher surgical trainees (HST). The $\mathrm{HST}^{\prime}$ 's rotated between the two hospital sites. Any trabeculectomy carried out in conjunction with an additional surgical procedure (eg cataract) was excluded. Patients who had undergone previous ocular surgery were included in this study.

All patients had a standard Cairn's type trabeculectomy. The trabeculectomy was performed with a fornix-based conjunctival flap, partial thickness scleral flap, full thickness sclerostomy, peripheral iridectomy, and fixed sutures. No adjustable sutures were used. A superior rectus stay suture was used and diathermy of sclera.

No alteration was made to the patient's systemic medication prior to or following surgery and no instruction was given to discontinue any medication. All patients had local anaesthesia in the form of peribulbar or subtenon's method and a minority had general anaesthesia. All intraocular pressures (IOPs) were measured using Goldmann's tonometry. Postoperatively all patients had topical steroid treatment for a minimum of 4 weeks.

The following was extracted from the case notes: (1) age of the patient, (2) type of glaucoma, (3) antiglaucoma medication, (4) use of anticoagulation or antiplatelet therapy, (5) use of antimetabolites, (6) postoperative treatment and haemorrhagic complications, (7) the presence of hyphaema on the first post-operative day, (8) return to theatre as a result of haemorrhagic complications, and (9) IOP during the first 2 years postoperatively and the use of antiglaucoma medication during this time. Surgical success was defined in two categories as an IOP of $<21$ and $<16 \mathrm{mmHg}$ with and without antiglaucoma medication 2 years following trabeculectomy. This criteria was used in accordance with the UK national trabeculectomy audit (NTA) performed at the same time as our study, that used and set similar standards for trabeculectomy outcome. ${ }^{9}$ A hyphaema was defined as a level of blood noted on the first postoperative day. All patients included in this study were examined on the first postoperative day.

All data were collected on standardised forms and entered into an Excel spreadsheet. Appropriate statistical tests were used including Student's $t$-test and Z-test.

\section{Results}

Data were collected from case notes in 82 and $92 \%$ of all trabeculectomies performed at the two hospital sites. A total of 367 trabeculectomies were analysed. All patients were Caucasian with a mean age at the time of surgery of 69.7 (8-88) years. The various classifications of glaucoma in the group were open-angle $(273,74.4 \%)$, narrow-angle $(31,8.4 \%)$, low-tension $(20,5.4 \%)$, pseudoexfoliation $(17,4.6 \%)$, uveitis $(3,0.8 \%)$, pigment dispersion $(3,0.8 \%)$, rubeotic $(3,0.8 \%)$, and other secondary and complex glaucoma $(17,4.6 \%)$ (Table 1$)$.

The mean presenting IOP was $30.78 \mathrm{mmHg}$ (14-74) for the whole group. The mean presenting IOP in those with a hyphaema was $30.83 \mathrm{mmHg}$ and the mean presenting IOP of those who did not have a hyphaema was $30.63 \mathrm{mmHg}$. This difference was not statistically significant ( $P=0.85$, Student's $t$-test, unpaired). The mean presenting IOP of those on aspirin was $28.39 \mathrm{mmHg}$ and this was significantly lower than those not taking aspirin $(31.31 \mathrm{mmHg})(P=0.04$, Student's t-test, unpaired).

Table 1 Types of glaucoma

\begin{tabular}{lrc}
\hline Type of glaucoma & Number & Percentage \\
\hline Primary open angle/juvenile open angle & 273 & 74.4 \\
Primary narrow-angle glaucoma & 31 & 8.4 \\
Low tension & 20 & 5.4 \\
Pseudoexfoliation & 17 & 4.6 \\
Fuchs heterochromic cyclitis & 3 & 0.8 \\
Rubeotic & 3 & 0.8 \\
Pigment dispersion & 3 & 0.8 \\
Others & 17 & 4.6 \\
Total & 367 & \\
\hline
\end{tabular}


Antiglaucoma medication history is outlined in Figure 1. A total of 140 patients $(38.2 \%)$ were on one topical medication, $172(46.9 \%)$ on two and 40 (10.9\%) on three topical medications prior to surgery. In the NTA, $50 \%$ of patients were on two medications at the time of listing for surgery and this compares closely with our study cohort. ${ }^{9}$ The average number of medications prior to surgery was 1.7. Figure 1 outlines the frequency of use of different antiglaucoma medications.

Six patients $(1.6 \%)$ had intraoperative antimetabolites. Five patients had application of 5-fluorouracil $(25 \mathrm{mg}$ / $\mathrm{ml}$ ) and of these one patient was on aspirin. One patient had application of mitomycin $\mathrm{C}(0.2 \mathrm{mg} / \mathrm{ml})$ and this patient was not on any APACT. In the NTA, $6.4 \%$ of patients had intraoperative antimetabolites. ${ }^{10}$ Three patients $(0.82 \%)$ had postoperative antimetabolites and of these, one patient was on warfarin. In the NTA, $2.8 \%$ of patients had postoperative antimetabolites.

In all, $55(15 \%)$ patients were on aspirin and five (1.4\%) on warfarin. None of the patients on aspirin suffered serious intra- or postoperative haemorrhage. Two cases of suprachoroidal haemorrhage were noted following fundoscopy in the postoperative period. One patient was on aspirin and the other patient was not on any APACT. Both cases showed spontaneous resolution. No cases in this group required return to theatre as a result of haemorrhagic complications.

One patient on warfarin had peribulbar anaesthesia and suffered a periorbital haematoma. Local anaesthesia was chosen as the patient had a degree of chronic obstructive airways disease. This was better controlled after additional medication and this patient together with three other patients on warfarin all had general

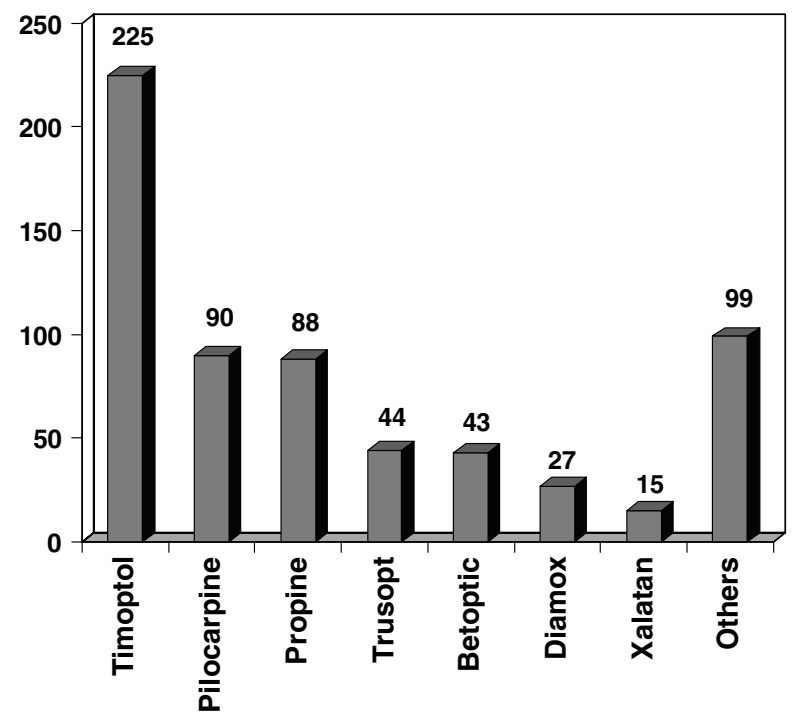

Figure 1 Frequency of preoperative use of different types of antiglaucoma medication. anaesthesia. One patient on warfarin had uneventful subtenon's anaesthesia. None of the five patients on warfarin had previous ocular surgery or intraoperative antimetabolites. All five patients developed significant hyphaema.

In all cases the therapeutic international normalised ratio (INR) was determined preoperatively and ranged from 1.5 to 4.5 . Case one developed $50 \%$ hyphaema on the first postoperative day. The patient was prescribed oral tranexamic acid $1.5 \mathrm{~g}$ tid for 2 weeks despite which the hyphaema took 3 weeks to resolve. The trabeculectomy failed within the first 6 months of surgery. Case two developed eight ball hyphaema on the second postoperative day. Medical advice was sought prior to surgery and a decision was made that it was unsafe to stop warfarin as the patient had a pig heart valve. The hyphaema was surgically evacuated 3 days following surgery and postoperatively the patient had two injections of $10 \mathrm{mg}$ of 5 -fluorouracil. After 6 years IOP remains controlled at $10 \mathrm{mmHg}$. Case three developed a small hyphaema postoperatively and was sent home 3 days after surgery. At 7 days after surgery the patient returned with vision of perception of light and $80 \%$ hyphaema. Surgical evacuation of the hyphaema was performed 13 days after surgery. No antimetabolites were used in the postoperative period. The trabeculectomy failed within 6 months of surgery. Cases four and five also developed significant hyphaema $(>30 \%)$ in the postoperative period taking more than 2 weeks for natural resolution. Neither of these cases had further intervention or surgery. Both cases failed within the 1st year of trabeculectomy. Within a year of trabeculectomy four patients in this group had IOPs in excess of $21 \mathrm{mmHg}$ and were placed on antiglaucoma medication.

Postoperatively 119 (32.4\%) patients developed hyphaema on the first postoperative day, including the warfarinised patients. The hyphaema rate was significantly higher in those on APACT $(33,55 \%)$, compared to those not on APACT $(86,28.0 \%)$ $(P=0.0001)$. The hyphaema rate was also significantly higher in those on aspirin $(28,50.9 \%)$ compared to those not on APACT $(P=0.0015)$. Two patients on aspirin had a transient rise in IOP in the presence of hyphaema. All hyphaemas cleared spontaneously with the exception of the two warfarinised patients outlined above (Table 2).

Surgical outcome is outlined in Tables 3 and 4 . The results were categorized into three groups of IOP ranges of $<16 \mathrm{mmHg}$, between 16 and $20 \mathrm{mmHg}$ and $\geq 21 \mathrm{mmHg}$ with (qualified) and without (unqualified) antiglaucoma medication 2 years following trabeculectomy. The warfarinised patients were excluded and the group was further divided and analysed into those with and without hyphaema and those with and 
Table 2 Preoperative topical antiglaucoma medications

\begin{tabular}{lcc}
\hline Number of medications & Frequency & Percentage \\
\hline One & 140 & 38.1 \\
Two & 172 & 46.9 \\
Three & 40 & 10.9 \\
Missing data & 15 & 4.1 \\
Total & 367 & \\
\hline
\end{tabular}

without aspirin. At every level of comparison the two

Table 3 Comparison of IOP at 2 years between 'hyphaema' and 'no hypahaema' groups

\begin{tabular}{|c|c|c|c|c|c|c|}
\hline \multirow{2}{*}{$\begin{array}{l}\text { Intraocular } \\
\text { pressure at } \\
2 \text { years }\end{array}$} & \multirow[t]{2}{*}{$\begin{array}{l}\text { Qualified }^{\mathrm{a}} \\
\text { or unqualified }\end{array}$} & \multicolumn{2}{|c|}{$\begin{array}{c}\text { Hyphaema } \\
\text { group }\end{array}$} & \multicolumn{2}{|c|}{$\begin{array}{l}\text { 'No hyphaema' } \\
\text { group }\end{array}$} & \multirow[t]{2}{*}{ P-value } \\
\hline & & $\mathrm{n}$ & $\%^{\mathrm{b}}$ & $\mathrm{N}$ & $\%^{\mathrm{b}}$ & \\
\hline \multirow[t]{2}{*}{$<16 \mathrm{mmHg}$} & Unqualified & 46 & 42.2 & 101 & 43.0 & 0.88 \\
\hline & Qualified & 2 & 1.8 & 11 & 4.7 & 0.13 \\
\hline \multirow[t]{2}{*}{$16-20 \mathrm{mmHg}$} & Unqualified & 39 & 35.8 & 80 & 34.0 & 0.76 \\
\hline & Qualified & 8 & 7.3 & 19 & 8.1 & 0.81 \\
\hline \multirow[t]{2}{*}{$\geq 21 \mathrm{mmHg}$} & Unqualified & 6 & 5.5 & 15 & 6.4 & 0.75 \\
\hline & Qualified & 8 & 7.3 & 9 & 3.8 & 0.21 \\
\hline Missing data & & 5 & & 13 & & \\
\hline Total number & & 114 & & 248 & & \\
\hline
\end{tabular}

aa Qualified' indicate only patients with antiglaucoma medication (excluding those without such medication.).

bPercentage is calculated after excluding the missing data from the total number and excluding the five patients on warfarin.

groups had a similar surgical outcome. There was no difference in surgical outcome between those who had a hyphaema and those that did not (Table 3). The overall success for an unqualified IOP of $<21 \mathrm{mmHg}$ was $78.0 \%$ in the hyphaema group and $77.0 \%$ in the nonhyphaema group. In the NTA $84.2 \%$ of patients achieved an unqualified IOP of $<21 \mathrm{mmHg}$. There was no difference in surgical outcome between patients on aspirin and those not on aspirin (Table 4).

Two patients on aspirin with low-tension glaucoma had autologous blood injected into the trabeculectomy bleb for clinical hypotony with no adverse events.

\section{Discussion}

The primary aim of our study was to ascertain the safety profile in continuing with anticoagulation during glaucoma surgery. Our data indicates that patients may safely continue on oral aspirin without suffering serious intra- and postoperative haemorrhagic complications in trabeculectomy.
Table 4 Comparison of IOP at 2 years between group of patients on aspirin with those not on any APACT

\begin{tabular}{|c|c|c|c|c|c|c|}
\hline \multirow{2}{*}{$\begin{array}{l}\text { Intraocular } \\
\text { pressure at } \\
2 \text { years }\end{array}$} & \multirow[t]{2}{*}{$\begin{array}{l}\text { Qualified }^{\mathrm{a}} \\
\text { or unqualified }\end{array}$} & \multicolumn{2}{|c|}{$\begin{array}{l}\text { 'Aspirin' } \\
\text { group }\end{array}$} & \multicolumn{2}{|c|}{$\begin{array}{l}\text { 'No APACT' } \\
\text { group }\end{array}$} & \multirow[t]{2}{*}{ P-value } \\
\hline & & $\mathrm{n}$ & $\%^{\mathrm{b}}$ & $\mathrm{n}$ & $\%^{\mathrm{b}}$ & \\
\hline \multirow[t]{2}{*}{$<16 \mathrm{mmHg}$} & Unqualified & 21 & 40.4 & 127 & 43.5 & 0.67 \\
\hline & Qualified & 3 & 5.8 & 10 & 3.4 & 0.49 \\
\hline \multirow[t]{2}{*}{$16-20 \mathrm{mmHg}$} & Unqualified & 17 & 32.7 & 104 & 35.6 & 0.68 \\
\hline & Qualified & 6 & 11.5 & 19 & 6.5 & 0.28 \\
\hline \multirow[t]{2}{*}{$\geq 21 \mathrm{mmHg}$} & Unqualified & 2 & 3.9 & 19 & 6.5 & 0.38 \\
\hline & Qualified & 3 & 5.8 & 12 & 4.1 & 0.63 \\
\hline Missing data & & 3 & & 15 & & \\
\hline Total number & & 55 & & 307 & & \\
\hline
\end{tabular}

a`Qualified' indicate only patients with antiglaucoma medication (excluding those without such medication.).

bercentage is calculated after excluding the missing data from the total number and excluding the five patients on warfarin.

We acknowledge that the retrospective nature of our study implies that there may not have been accurate documentation of troublesome intraoperative bleeding during trabeculectomy. The study included patients operated on by a number of surgeons including HST. Small variations in technique including the amount of diathermy used may have influenced the degree of intraoperative bleeding. However, this complication would have been difficult to quantify and certainly did not lead to any haemorrhagic complications that necessitated return to theatre. The retrospective nature of the study also did not allow us to take into account any patients that may have been on Ginkgo biloba which has been incriminated as a cause of spontaneous

hyphaema. ${ }^{11}$ However, we believe the number of such patients, if any, would be too low to have a significant effect on the results.

Patients on aspirin had nearly twice the risk of developing hyphaema $(50.9 \%)$ compared to those that were not on aspirin $(28.0 \%)$. The hyphaema rate in aspirin is high even when compared to the NTA that had a hyphaema rate of $24 \% .{ }^{12}$ A hyphaema in the early postoperative period may lead to a transient rise in IOP and visual obscuration. It may be useful to include these outcomes when consenting patients for trabeculectomy.

Patients on warfarin may suffer serious haemorrhagic complications with trabeculectomy despite the INR being within the therapeutic range. The early visual loss experienced by patients in the postoperative period can be quite alarming. In our study, all five patients developed significant hyphaema, two of whom required 
surgical evacuation. The only case that had long-term success was case two with early surgical evacuation of hyphaema and postoperative antimetabolites.

Our data indicates that patients on warfarin require extreme caution during trabeculectomy. Preoperatively a thorough medical assessment is required which should include blood pressure, INR, drug interactions, and possible temporary heparinisation, all of which may minimise the risk of bleeding. The minimum period required in order to alter coagulation status before surgery is 3 days for warfarin. ${ }^{5}$ Intraoperatively meticulous surgical technique may also minimise the risk of bleeding. Konstas and Jay ${ }^{13}$ showed that the site of trabeculectomy fistula had a profound effect on the hyphaema rate and that the more anterior the fistula the less the chance of a hyphaema. They postulated that the vascular components of the sclera and ciliary body were the most likely sources of blood. Parkin and Manners ${ }^{5}$ suggested that anticoagulated patients undergoing ophthalmic surgery should have individualised preoperative protocols, close liaison with medical and haematological colleagues, heparinisation and finally convincing evidence as to the value of surgery given the risks involved.

In the event of significant hyphaema our experience suggests that early evacuation of blood is probably the best option for long-term survival of the trabeculectomy bleb. Blood is a well-known mediator of inflammation and indeed injections of blood are used to reduce aqueous drainage in hypotonous eyes. ${ }^{14}$ In large amounts and possibly not only in the anterior chamber but also in the trabeculectomy ostium and bleb subconjunctival tissue, blood would mediate conjunctival scarring and trabeculectomy failure. The haemorrhagic tendency in warfarinised patients and subsequent risk of bleb failure may be an indication for the use of intraoperative antimetabolites. The failure of the trabeculectomy in four cases indicates that these patients may benefit from close monitoring in the postoperative period with a more intense regime of postoperative steroids and possible bleb intervention.

Other studies have also shown that anticoagulated patients may suffer catastrophic bleeding during ophthalmic surgery. Narendran and Williamson studied 541 patients undergoing vitreoretinal surgery and concluded that aspirin had little effect on significant perioperative haemorrhage. Seven patients on warfarin on the other hand had a statistically significant association with bleeding complications and the authors recommended that warfarin should be stopped prior to vitreoretinal surgery providing the thromboembolic risk was low. ${ }^{15}$

The UK NTA audited trabeculectomies performed in 1996 which coincides with the midpoint of our study period and provides a standard against which glaucoma surgery in individual departments could be compared. There was little use of antimetabolites during that period and one of their criteria for surgical success was an IOP of $<21 \mathrm{mmHg}$ a year after trabeculectomy without the aid of antiglaucoma medication. Using this criterion the NTA achieved a success rate of $84 \%$. For similar criteria our success rate was $77 \%$. We feel our success rate was lower because our study cohort included complicated glaucomas, patients who had previous ocular surgery and a follow-up period of 2 years, all of which contribute towards increased trabeculectomy failure. It is reassuring that all groups overall whether those with or without hyphaema as well as those with and without aspirin when looked at separately had a similar surgical outcome 2 years following trabeculectomy in terms of IOP control. We can now safely inform patients that a hyphaema does not necessarily affect the long-term outcome of IOP control in those taking aspirin.

In conclusion aspirin appears safe to continue with during trabeculectomy. Hyphaema is more common in patients on aspirin. This has no significant adverse shortor long-term effect. IOP control is unaffected by early hyphaema, 2 years after trabeculectomy. Warfarin is associated with serious haemorrhagic complications. Patients require careful individual monitoring in the pre- and postoperative period and are at risk of trabeculectomy failure.

\section{Acknowledgements}

We thank Dr Alan Cox, Department of Ophthalmology, Ninewells Hospital and Medical School and Ms Gwen Bayne, Queen Margaret Hospital for their invaluable assistance with the statistics.

\section{References}

1 Dalen JE. An apple a day or an aspirin a day? Arch Int Med 1991; 151: 1066-1068.

2 Dick B, Jacobi FK. Cataract surgery and anticoagulation therapy. Klin Monatsbl Augenh 1996; 209: 340-346.

3 Eke T, Thompson JR. The national survey of local anaesthesia for ocular surgery 2. Safety profiles of local anaesthesia techniques. Eye 1999; 13: 196-204.

4 Stone LS, Kline Jr OR, Sklar C. Intraocular lenses and anticoagulation and antiplatelet therapy. Am Intra-Ocul Implant Soc 1985; 11: 165-168.

5 Parkin B, Manners R. Aspirin and warfarin therapy in oculoplastic surgery. Br J Ophthalmol 2000; 84: 1426-1427.

6 Flaxel CJ, Blach RK. Aspirin is a hazard for vitreoretinal surgery. Br J Ophthalmol 1996; 80: 486.

7 Bell NP, Orengo-Nania S, Pietz K, Gross RL. Aspirin use in advanced uncontrolled glaucoma. J Glaucoma 2004; 13(5): 365-370. 
8 McCormack P, Simcock PR, Tullo AB. Management of the anticoagulated patient for ophthalmic surgery. Eye 1993; 7: 749-750.

9 Edmunds B, Thompson JR, Salmon JF, Wormald RP. The national survey of trabeculectomy 1 . Sample and methods. Eye 1999; 13: 524-530.

10 Edmunds B, Thompson JR, Salmon JF, Wormald RP. The national survey of trabeculectomy 2. Variations in operative technique and outcome. Eye 2001; 15: 441-448.

11 Rosenblatt M, Mindel J. Spontaneous hyphema associated with ingestion of Ginkgo biloba extract. N Engl J Med 1997; 336: 1108 .
12 Edmunds B, Thompson JR, Salmon JF, Wormald RP. The national survey of trabeculectomy 3. Early late complications. Eye 2002; 16: 297-303.

13 Konstas AGP, Jay JL. Modification of trabeculectomy to avoid postoperative hyphaema. The 'guarded anterior fistula' operation. Br J Ophthalmol 1992; 76: 353-357.

14 Smith MF, Magauran III RG, Betchkal J, Doyle JW. Treatment of postfiltration bleb leaks with autologous blood. Ophthalmology 1995; 102(6): 868-871.

15 Narendran N, Williamson TH. The effects of aspirin and warfarin therapy on haemorrhage in vitreoretinal surgery. Acta Ophthalmol Scand 2003; 81: 38-40. 